\title{
Genotypic identification of extended spectrum $\beta$-lactamase producing Escherichia coli in dairy supply chain
}

\author{
Amarjeet Kumar ${ }^{1}$, Rashmi Hogarehalli Mallappa ${ }^{2}$, Avinash Jaswal ${ }^{1}$, Brijesh Kumar ${ }^{1}$, Naresh Kumar ${ }^{1}$ and Raghu Hirikyathanahalli \\ Vishweswaraiah $^{1}$
}

Received: 02 November 2020 / Accepted: 06 December 2020 / Published online: 28 February 2021

(C) Indian Dairy Association (India) 2021

\begin{abstract}
Foodborne illnesses due to antibiotic-resistant bacteria represents a major public health problem in both developed and developing countries. Among 190 samples, 139 Escherichia coli positive isolates from raw milk, pasteurized milk, and human handlers were identified by phenotypic methods and genotypic methods. All E. coli isolates were found to be resistance to penicillin, oxacillin, erythromycin and clindamycin. The dominant type of resistance to cefotaxime and amoxiclave identically detected in $18.7 \%$ isolates followed by ampicillin in $17.98 \%$, trimethoprim $15.82 \%$, tetracycline $10.79 \%$, nalidixic acid $7.91 \%$, and piperacillin $7.79 \%$. Four isolates have shown resistance $(2.87 \%)$ to Ceftriaxone and ceftazodime, Cefotaxime and one isolate has shown resistance to Cefepime. Further, all four isolates were confirmed as extended spectrum $\beta$-lactamase (ESBL) producer by double disc diffusion test and ESBL chromogenic medium. Later, all four isolates were evaluated by PCR and they are observed as carrier of blaCTX $\mathrm{M}$ gene which is responsible for ESBL antibiotic resistance in E.coli but blaTEM and blaSHV genes were absent in all four ESBL isolates. Based on the above findings, it is concluded that ESBL antibiotic resistance in E. coli were more prevalent in milk and this may due to spread and acquirement of antibiotics resistance gene by plasmid and mobile genetic elements.
\end{abstract}

Keyword: Antibiotic resistance, E. coli, Milk, ESBL

\footnotetext{
${ }^{1}$ National Referral centre, Dairy Microbiology Division, ICAR-NDRI, Karnal-132001. Haryana, India

${ }^{2}$ Molecular Biology Unit, Dairy Microbiology Division, ICAR-NDRI, Karnal-132001

Raghu Hirikyathanahalli Vishweswaraiah $(\triangle)$

National Referral Centre, Dairy Microbiology Division,

ICAR-National Dairy Research Institute, Karnal-132001. Haryana, India.

Email: raghu.nrcndri@yahoo.com ;4rvsy.dmndri@gmail.com

Tel: +91-184-2259534, Mobile: +91-9466963599
}

\section{Introduction}

The enzyme responsible for degradation of oxyimino- $\beta$-lactam antibiotics is Extended-spectrum $\beta$-lactamases (ESBL) and these antibiotics are used in the treatment of various serous humans and animal infections (Palmeira and Ferreira, 2020). ESBL were first identified in the year 1983 in the family Enterobacteriaceae and they are responsible for serious risk to human health may be due to treatment failure in various severe cases of infections in 2013 (Adeolu et al. 2016). These enzymes are encoded by plasmids that confer resistance to the penicillins; to first-, second-, third, and fourth-generation cephalosporin's; and to aztreonam but not to carbapenems (EFSA, 2011). Human-to-human spread of these type of enzyme producing bacterial pathogens have been demonstrated in public hospital settings, representing that human colonization is a pool for dissemination (Liebana et al. 2013). Furthermore, various reports are available for the isolation and identification of ESBL-producing bacteria from foods and food animals, suggesting the likely role of the food production chain as a pool for this group of pathogenic bacteria (EFSA, 2019, Odenthal et al. 2016). There are various data's are available regarding robust correlation between the incidence of ESBLproducing bacteria in foods (Algeria et al. 2020) and the prevalence of infections in humans may be anticipated that food obtained from animals may be infected with ESBL producers which contributing to the transmission within the population (EFSA, 2011). Furthermore, ESBL producers also can the resistant bacteria can fetch additional genes having some virulence property; it is notable that strains of Escherichia coli (STEC) are known to be food-borne pathogens, have been confirmed as ESBL-producers, representing that the transference of the extracellular DNA from commensals to foodborne pathogenic strains is possible (Torpdahl et al. 2013).

The occurrence of ESBL-producing Escherichia coli is extensively growing throughout India. These pathogens pose a major threat to the treatment of infection and may cause tricky in the management of infections. This may create unnecessary problem with the widespread use of second- or third-generation antibiotics for the monitoring and treatment of bacterial infections (Van Hoek et al. 2015). ESBL E. coli is typically unaffected to routinely used antibiotics instigating a surge in the use of almost 
all antimicrobials (i.e., carbapenems) in treatment. The $E$. coli strains harboring those resistance genes can easily spread to other pathogens leading to the spread of resistance (Odenthal et al. 2016). Hence, the presence of ESBL-producing E. coli in the dairy supply chain maybe arriving from healthy animals is the fact which has to be suitably premeditated. Therefore, in our present study, we are evaluating the occurrence of ESBLproducing $E$. coli in dairy supply chain.

\section{Materials and Methods}

\section{Procurement and Maintenance of culture}

The standard culture used in our study was Escherichia coli ATCC 25922 was purchased from American Type Culture collection. The culture was activated in nutrient broth followed by streaking on Violet red bile (VRBA) agar followed by incubation for overnight at $37^{\circ} \mathrm{C}$. A single pure colony from VRBA after microscopic examination was picked up and maintained on nutrient agar slant by routine sub-culturing after every fortnight. All the experiments were conducted using overnight grown cultures. All media chemicals including Muller Hinton Agar and antimicrobial agents including discs were procured from Himedia lab (Mumbai, India).

\section{Detection of $E$. coli using PCR}

Species specific primers were used in Colony polymerase chain

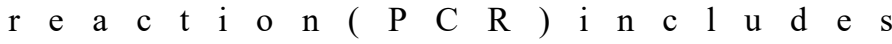
Forward:GGTAACGTT T C TACCGCAGAGTTG/ Reversed:CAGGGTTGGTACACTGTCATTACG, target gene of $E$. coli as phoA with a size of 468 bp (Shome et al. 2011) using a protocol given by Godambe et al. (2017).

\section{Antimicrobial susceptibility tests (AST)}

Antimicrobial susceptibility tests was carried out on MuellerHinton Agar (Hi-media, Mumbai, India) using the Kirby-Bauer disk diffusion method (Bauer et al. 1996). The data were evaluated and inferred according to National Committee for Clinical Laboratory Standards (NCCLS 1993). Reference strain of E. coli ATCC 25922 was used as a quality control strain for studying AST (CLSI 2007). The resistance strains were evaluated for minimum inhibitory concentration (MIC) using micro-dilution methods followed data inferred as per CLSI guidelines (CLSI 2007).

\section{Extended Spectrum $\beta$-lactamase (ESBL) confirmatory test}

The double disc synergy test (DDST) was performed for ESBL using amoxicillin/clavulanate, ceftazidime, ceftriaxone, aztreonam, and cefotaxime (Jarlier et al. 1988; Drieux et al. 2008). Further, phenotypic confirmation of ESBL positive isolates was carried out using CLSI (2012) guidelines. The test is considered as positive when a decreased susceptibility to cefotaxime is combined with a clear-cut enhancement of the inhibition zone of cefotaxime in front of the clavulanate-containing disk, often resulting in a characteristic shape-zone referred to as 'champagne-cork' or 'keyhole (Drieux et al. 2008). A ratio of ceftazidime or cefotaxime MIC to ceftazidime or cefotaximeclavulanic acid MIC equal to or greater than eight indicated the presence of ESBL (Drieux et al. 2008).

\section{Phenotypic identification of ESBL in E. coli}

An overnight grown culture of ESBL + ve isolate whose turbidity was adjusted to $0.5 \mathrm{McF}$ arland solutions (Himedia Lab, Mumbai, India) was streaked on the Hicrome ESBL agar plates followed by incubation at $37^{\circ} \mathrm{C}$ for $24 \mathrm{~h}$. The development of pink or purple colored colonies on the Hicrome ESBL agar plates considered as positive for ESBL.

\section{Identification of ESBL by PCR}

The following ESBLs resistance determinants were investigated by PCR for bla-CTXM, bla-TEM and bla-SHV gene (Karczmarczyk et al. 2011). Colony PCR were performed as per protocol given by Godambe et al. (2017) by targeting ESBL encoding genes [Primer Sequence (5'-3') such as CTX-M universal- FCGATGTGCAGTACCAGTAA; CTX-M universal-RT T A G T G A C C A G A A T C A G C G G, b l a S H V - F T T A T C T C C C T G T T A G C C A C C; b l a S H V - R GAT T T GCT GAT T T C GC T C G G, b la T E M - F G C G G A A C C C C T A T T T G a n d b la T E M - R ACCAATGCTTAATCAGTGAG] (Olesen et al. 2004) with a genome size of $585 \mathrm{bp}$ for CTX-M (Batchelor et al. 2005) and 795 bp for blaSHV (Weill et al. 2004).

\section{Results and Discussion}

\section{Detection of $E$. coli in dairy supply chain}

In our study, the prevalence of $E$. coli in raw milk samples were found to be $57.27 \%, 20 \%$ in pasteurized milk samples, and $25 \%$ in swab of human handler working in dairy farms and plants by biochemical identification test such as Indole, Methyl red, Vogues Prausker test and citrate utilization test. From the above, 139 isolates were selected and confirmed as E. coli using specific enzyme substrate interaction using two stage enzyme assay and Hichrome ECD agar wherein all isolates have shown characteristic enzyme reaction and characteristic blue colored colonies on Hichrome ECD agar (Fig. 1). Later, these isolates were shown characteristic $468 \mathrm{bp}$ amplicon bands on gelelectroporesis based on the use of species specific primer. The amplification was seen in the reference strain E. coli ATCC 25922 which was used as positive control. Almost similar prevalence rate of $E$. coli were reported by Soomro et al. (2002) wherein they found 57 and 52\% prevalence of $E$. coli in raw milk respectively. Tadesse et al. (2012) and Badri et al. (2017) have shown lower prevalence rate (33\%, $38 \%$ ) of $E$. coli in raw milk samples respectively. The variation in 


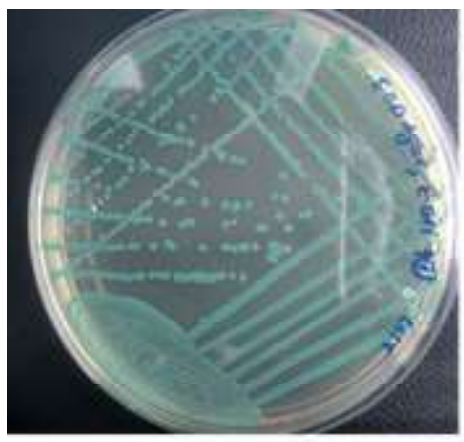

Gir-9-1 E. coll

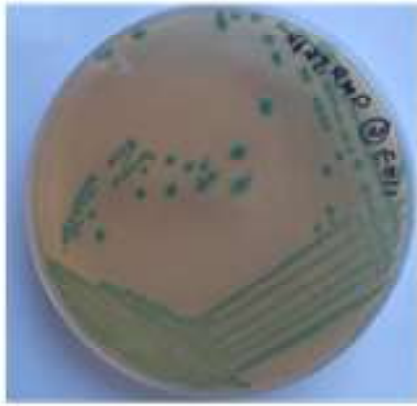

Kt27RMD-2

E. coli

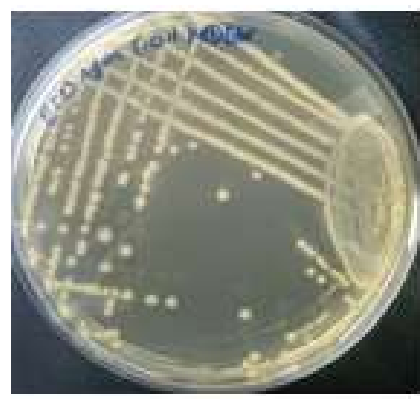

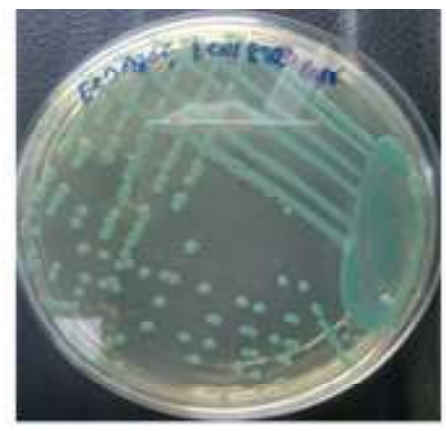

Gir-83-1 E. coli

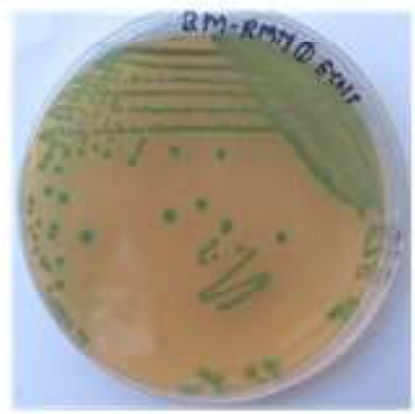

BM-RM-7-1

E. coli

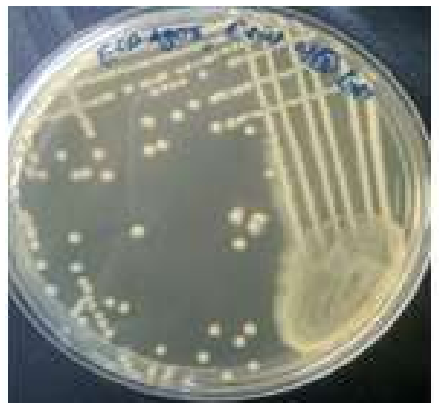

Negative control

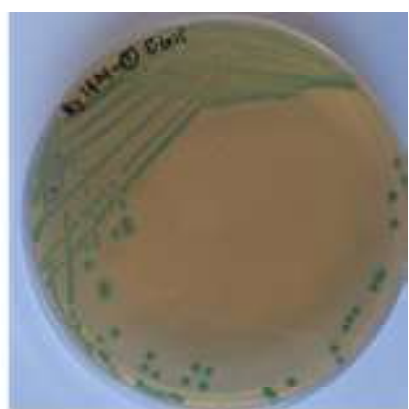

Kj14M-1 E. coll

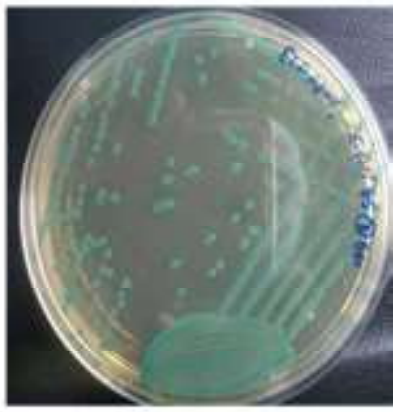

Gir-85-2 E. coli

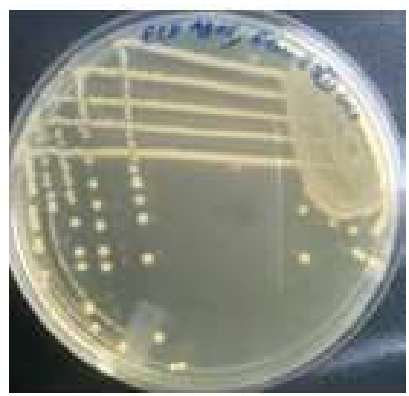

Fig. 1. Rapid detection of E. coli isolates on Hichrome ECD/MUG agar

prevalence rate of E. coli may be attributed to lapses in clean milk production, different geographic location and season, fecal contamination of milk, and due to poor hygiene and sanitary practices followed while milking and further handling (Thaker et al. 2013). The prevalence of E.coli in pasteurized milk may be due to post processing contamination and poor hygienic management subsequently the milk is pasteurized (Ali and Abdelgadir, 2011)

\section{Antibiotic susceptibility test}

Among 139 E. coli isolates, all isolates have shown resistance towards penicillin $(\mathrm{P})$, oxacillin $(\mathrm{OX})$ and erythromycin $(\mathrm{E})$, respectively based on inhibition zone diameter by AST followed by 26 isolates have shown resistance towards amoxicillin (MC) and Cefotaxime (CTX), 25 isolates to ampicillin (AMP), 22 isolates to trimethoprim (TR), 15 isolates to tetracycline (TET), 11 isolates to nalidixic acid (NA), 10 isolates to piperacin (PI), 5 isolates to gentamycin (GEN) and chloramphenicol (C), 4 isolates ceftriaxone (CTR) and cefepime (CPM), 3 isolates to nitrofurantoin (NIT) and amikacin (AK), 1 isolate has shown resistance towards ceftazidime (CAZ) and ofloxacin (OF), and none of the isolates have shown resistance towards ampicillin-sulbactam (A/S), meropenem (MRP), ertapenem (ERP) and netilmicin (NET), respectively (Table 1). Based on the AST, it was clear that four isolates were showed resistance towards ESBL and none were showing resistance to carbapenem group of antibiotics. The 
Table 1 Prevalence of antibiotics resistant pattern of $E$. coli

\begin{tabular}{lllc}
\hline Name of antibiotics & No of Resistance isolates & $\begin{array}{l}\text { No of Intermediate } \\
\text { isolates }\end{array}$ & No of Susceptible isolates \\
\hline P & $139(100 \%)$ & 0 & 0 \\
AMP & $25(17.98)$ & $1(0.71 \%)$ & $113(81.29 \%)$ \\
OX & $139(100 \%)$ & 0 & 0 \\
PI & $10(7.19 \%)$ & $16(11.51 \%)$ & $113(81.29 \%)$ \\
AMC & $26(18.7 \%)$ & $40(28.77 \%)$ & $73(52.51 \%)$ \\
A/S & 0 & $8(5.75 \%)$ & $131(94.24 \%)$ \\
CTX & $4(2.87 \%)$ & $35(25.17 \%)$ & $100(71.94 \%)$ \\
CPM & $4(2.87 \%)$ & $35(25.17 \%)$ & $100(71.94 \%)$ \\
CTR & $4(2.87 \%)$ & 0 & $135(97.12 \%)$ \\
CAZ & $1(0.71 \%)$ & $8(5.75 \%)$ & $130(93.52 \%)$ \\
NA & $11(7.91 \%)$ & $24(17.26 \%)$ & $104(74.82 \%)$ \\
CIP & $2(1.43 \%)$ & $4(2.87 \%)$ & $133(95.68 \%)$ \\
OF & $1(0.71 \%)$ & 0 & 138 \\
TR & $22(15.82 \%)$ & $1(0.71 \%)$ & $116(83.45 \%)$ \\
C & $5(3.59 \%)$ & $2(1.43 \%)$ & $132(94.96 \%)$ \\
NIT & $3(2.15 \%)$ & 0 & $136(97.84 \%)$ \\
CD & $139(100 \%)$ & 0 & 0 \\
TE & $15(10.79 \%)$ & $3(2.15 \%)$ & $121(87.05 \%)$ \\
E & $139(100 \%)$ & 0 & 0 \\
AK & $3(2.15 \%)$ & $6(17.26 \%)$ & $112(80.57 \%)$ \\
GEN & $5(3.59 \%)$ & 0 & $128(92.08 \%)$ \\
MRP & 0 & 0 & $139(100 \%)$ \\
ETP & 0 & 0 & $139(100 \%)$ \\
NET & 0 & $139(100 \%)$ \\
\hline P.P & 0 & $0.31 \%)$ &
\end{tabular}

P: Penicillin-G, AMP: Ampicillin, OX:Oxacillin, PI: Piperacin, AMC: Amoxicillin, A/S: Ampicillin-sulbalactam, CTX: Cefotaxime, CPM: Cefepime, CTR Ceftriaxone, CAZ: Ceftazidime, NA: Nalidixic Acid, CIP: Ciprofloxacin, OF: Oflaxacillin, TR: Trimethoprim, C: Chloramphenicol, NIT: Nitrofurantoin, CD: Clindamycin, TE: Tetracycline, E: Erythromycin, AK: Amikacin, GEN: Gentamycin, MRP: Meropenam, ETP: Erthaapenem, NET: Netilimycin

dominant types of resistance was observed in our results are in close association with the result of Rasheed et al. (2014) wherein he was reported $14.7 \%$ of the isolate from raw milk were showing resistance towards ESBL. This may be due acquiring of mobile genetic element such as plasmids, transposons, and Class 2 integrons (Singh et al. 2005).

\section{ESBL $E$. coli}

Based on diameter of zone of inhibition by AST methods four isolates of $E$. coli have found positive for ESBL. Further, these 4 positive isolates were confirmed phenotypically as ESBL using double disk diffusion test (DDDT) and Hi-Chrome ESBL agar base (Fig. 2). All four ESBL positive isolates have shown an inhibition zone diameter of $\leq 27 \mathrm{~mm}$ for CTX and CTR, followed by three isolates have shown a inhibition zone diameter of $\leq 22$ $\mathrm{mm}$ for CAZ and CPM (Table 2). Overall prevalence of ESBL positive $E$. coli in raw milk samples was $3.27 \%$ No ESBL positive isolates were obtained from pasteurized milk and human handlers swab samples. All the ESBL positive isolates by DDDT and
Chromogenic ESBL medium have shown identical resistance towards (P, AMP, PI, CTX, CTR, CAZ, and CPM) and (P, PI, CTR, CPM, TE) by 4 and 2 E. coli isolates, respectively. Duan et al. (2006) reported a 3.1\% prevalence of ESBL producers among $E$. coli isolates from dairy cattle. In a Turkish study reported by Kucukbasmaci et al. (2008) reported 2.1\% prevalence of ESBL producing Enterobacteriaceae isolated from dairy cattle. Gundogan and Avci (2013) reported 10\% (2/20) prevalence of ESBL positive $E$. coli in milk which is slightly higher than current study. The prevalence rate of ESBL producing $E$. coli was $29.3 \%$ $(17 / 22)$ in raw milk which is much higher than the current study (Badri et al. 2017).

\section{PCR identification of ESBL in $E$. coli}

All four ESBL positive isolates were further confirmed as ESBL E.coli by genotypic methods using colony PCR wherein all 4 isolates have shown bands for bla CTXM gene yielded $885 \mathrm{bp}$ amplicon on agarose gel (Fig. 3). However, no amplified products were obtained with bla-SHV and bla-TEM primer. This indicates 

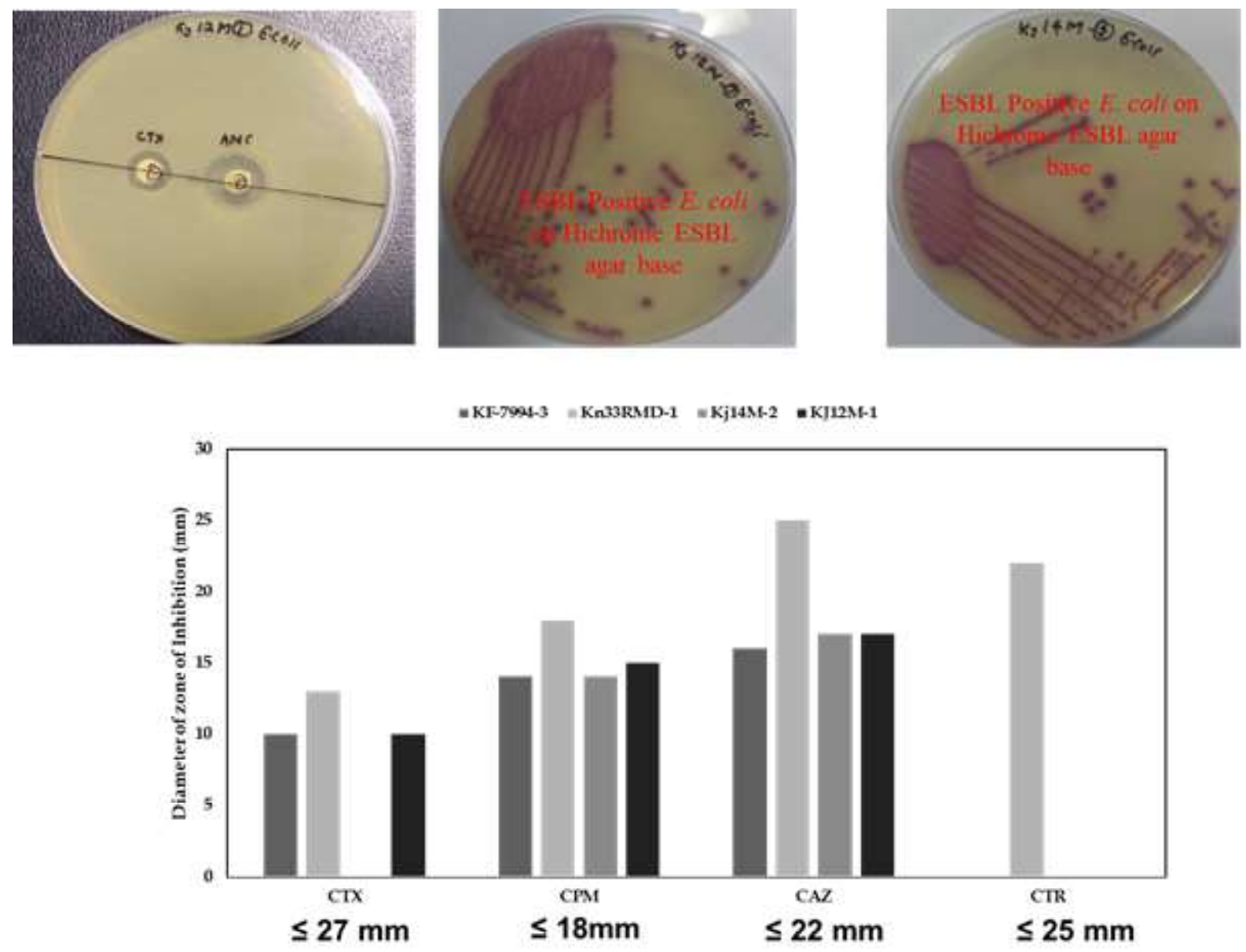

Fig. 2 Confirmation of ESBL + ve E.coli isolates by DDDT and ESBL chromogenic agar. a. double disc diffusion test b. ESBL +ve $E$. coli on Hicrhome ESBL agar c. Diameter of zone of inhibition in ESBL + ve silates by DDDT.

Fig. 3 ESBL producing isolates with primers specific for bla-CTXM and blaSHV gene. Lane 1 to 4 : ESBL producing isolates with bla-CTXM primer; Lane 5 to 9: ESBL producing isolates with blaSHV primer; Lane 10: Negative control for bla-CTXM primer; Lane 11: Negative control for bla-SHV primer

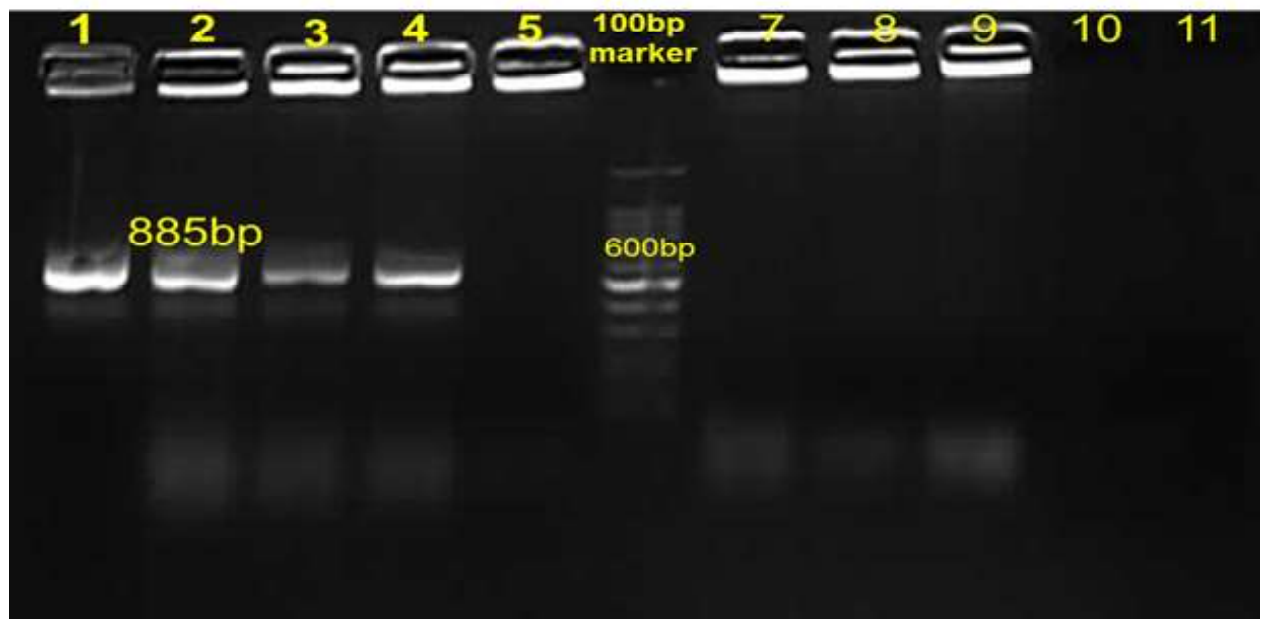

that all four ESBL producing isolates were harboring bla-CTX-M gene which encodes ESBL in $E$. coli isolates. Similar findings were reported by Batabyal et al. (2018) regarding the prevalence

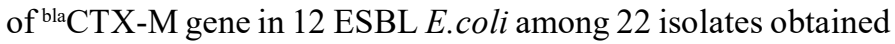
from West Bengal. Ghatak et al. (2013) have reported a one isolate was harboring New Delhi metallo $\beta$ lactamase gene $\left(b l a_{\mathrm{NDM}}\right)$ and another isolate was carrying ESBL gene - $b l a_{\text {Стх }}$. Further, Dhara and Tripathi (2014) has reported ESBL E.coli were found positive for bla CTX M-3 gene (18 nos), bla CTX M-9 gene (6 nos), bla SHV gene: (5 nos) and bla TEM gene: (5 nos) and may cause 
health risk to consumers due to contamination by ESBL producing $E$. coli, their pathogenicity and treatment failure as a result of antibiotic resistant.

\section{Conclusions}

Based on the above findings it is concluded that four $E$. coli isolates have shown resistance to ESBL antibiotics like CTX, CTR, CAZ and CPM may indicate presence of multiple drug resistance gene on same mobile genetic elements. Further, all four ESBL positive E. coli isolates were harboring CTX-M gene which is linked with dairy animal. It also concluded that the prevalence of ESBL $E$. coli in raw milk may due to transmission and acquisition of antibiotics resistance gene by plasmid and mobile genetic elements.

\section{Acknowledgment}

AK is the recipient of Junior Research Fellow scholarship from Indian Council of Agriculture Research, DARE, and Government of India. Director, ICAR-NDRI, Deemed University for furnishing the required research facility for conducting the study on AMR in E. coli. Indian Network Fishery and Animals antimicrobial resistance (INFAAR), ICAR and FAO are highly acknowledged for their help in undergoing a training program on antimicrobial resistance in bacterial pathogens. The project was initially supported by Science and Engineering Research Board, DST, GOI, New Delhi under the Grant number: SERB/ECR/2017/002333.

\section{References}

Adeolu M, Alnajar S, Naushad S, Gupta RS (2016) Genome-based phylogeny and taxonomy of the 'Enterobacteriales': proposal for Enterobacterales ord. nov. divided into the families Enterobacteriaceae, Erwiniaceae fam. nov., Pectobacteriaceae fam. nov., Yersiniaceae fam. nov., Hafniaceae fam. nov., Morganellaceae fam. nov., and Budviciaceae fam. nov. Int J Syst Evol Microbiol 66: 5575-5599

Alegría Á, Arias-Temprano M, Fernández-Natal I, Rodríguez-Calleja JM, García-López ML, Santos Jan (2020) Molecular diversity of ESBLproducing $E$. coli from foods of animal origin and human patients. Int J Environ Res Public Health 17:1312.

Ali AA, Abdelgadir WS (2011) Incidence of Escherichia coli in raw cow's milk in Khartoum state, British. J Dairy Sci 2:23-26

Badri AM, Ibrahim IT, Mohamed SG, Garbi MI, Kabbashi AS (2017) Prevalence of Extended Spectrum $\beta$-Lactamase (ESBL) Producing E. coli and Klebsiella pneumonia Isolated from Raw Milk Samples in Al Jazirah State, Sudan. Mol Biol 7: 2

Batabyal K, Banerjee A, Pal S, Dey S, Joardar SN, Samanta I, Isore DP, Singh AD (2018) Detection, characterization, and antibiogram of extended-spectrum $\beta$-lactamase $E$. coli isolated from bovine milk samples in West Bengal, India. Vet World 10:1423

Batchelor M, Hopkins K, Threlfall EJ, Clifton-Hadley FA, Stallwood AD, Davies RH, Liebana E (2005) blaCTX-M genes in clinical Salmonella isolates recovered from humans in England and Wales from 1992 to 2003. Antimicrob Agents Chemother 49: 1319-1322
Bauer AW, Kirby WMM, Sherris JC, Turck M (1966) Antibiotic susceptibility testing by a standardized single disk method. Am J Clin Pathol 45: 493-496

CLSI (2007 Performance standards. In: Institute CaLS, editor. M100-S17. vol. ISBN 1-56238-625-5, 1 edn. 940 West Valley Road, Suite 1400, Wayne 19087-1898 USA; 2007.

CLSI (2012) Performance standards for antimicrobial susceptibility testing. Clinical and Laboratory Standards Institute (M100eS22). 2012 (s22nd Informational Supplement).

Dhara L, Tripathi A (2014) Genetic and structural insights into plasmidmediated extended-spectrum $\beta$-lactamase activity of CTX-M and SHV variants among pathogenic Enterobacteriaceae infecting Indian patients. Int J Antimicrob Agents 43: 518-26.

Drieux L, Brossier F, Sougakoff W, Jarlier V (2008) Phenotypic detection of extended spectrum $\beta$ lactamase production in Enterobacteriaceae: review and bench guide. Clin Microbiol Infect 14: 90-103.

Duan RS, Sit TH, Wong SS, Wong RC, Chow KH, Mak GC, Ho PL (2006) Escherichia coli producing CTX-M $\beta$-lactamases in food animals in Hong Kong. Microb Drug Resist 12: 145-148

EFSA (2011) BIOHAZ Panel Scientific Opinion on the public health risks of bacterial strains producing extended-spectrum $\beta$-lactamases and/ or AmpC $\beta$-lactamases in food and food-producing animals. EFSA $J$ 9: 2322

EFSA (2019) ECDC. The European union summary report on antimicrobial resistance in zoonotic and indicator bacteria from humans, animals and food in 2017. EFSA J 17: 5598.

Ghatak S, Singha A, Sen A, Guha C, Ahuja A, Bhattacharjee U, Das S, Pradhan NR, Puro K, Jana C, Dey TK (2013) Detection of New Delhi Metallo $\beta$ Lactamase and Extended Spectrum $\beta$ Lactamase genes in E. coli isolated from mastitic milk amples. Transbound Emerg Dis 60: 385-389

Godambe LP, Bandekar J, Shashidhar R (2017) Species specific PCR based detection of Escherichia coli from Indian foods. 3 Biotech 7: 1-5

Gundogan N, Avci E (2013) Prevalence and antibiotic resistance of extended-spectrum $\beta$-lactamase (ESBL) producing $E$. coli and Klebsiella species isolated from foods of animal origin in Turkey. Afr J Microbiol Res 7: 4059-4064

Jarlier V, Nicolas MH, Fournier G, Philippon A (1988) Extended broadspectrum $\beta$-lactamases conferring transferable resistance to newer $\beta$-lactam agents in Enterobacteriaceae: hospital prevalence and susceptibility patterns. Clin Infect Dis 10: 867-878

Karczmarczyk M, Abbott Y, Walsh C, Leonard N, Fanning S (2011) Characterization of multidrug-resistant Escherichia coli isolates from animals presenting at a university veterinary hospital. Appl Environ Microbiol 77: 7104-7112

Kucukbasmaci O, Ciftcioglu G, Midilli K, Issa G (2008) Detection of extended spectrum $\beta$-lactamase producing Enterobacteriaceae from food animals In Turkey. Revue Méd Vét 159: 586-592

Liebana E, Carattoli A, Coque TM, Hasman H, Magiorakos AP, Mevius D, Peixe L, Poirel L, Schuepbach-Regula G, Torneke K (2013) Public health risks of enterobacterial isolates producing extended-spectrum $\beta$-lactamases or AmpC $\beta$-lactamases in food and food producing animals: An EU perspective of epidemiology, analytical methods, risk factors, and control options. Clin Infect Dis 56: 1030-1037

NCCLS (National Committee for Clinical Laboratory Standards) (1993) Tentative Guidelines, M26-TNCCLS. Villanova, PA: 1993. Methods for determining bactericidal activity of antimicrobial agents

Odenthal S, Akineden Ö, Usleber E (2016) Extended-spectrum $\beta$-lactamase producing Enterobacteriaceae in bulk tank milk from German dairy farms. Int J Food Microbiol 238: 72-78

Olesen I, Hasman H, Møller Aarestrup F (2004) Prevalence of $\beta$-lactamases among ampicillin-resistant $E$. coli and Salmonella isolated from food animals in Denmark. Microb Drug Resist 10: 334-340 
Palmeira DJ, Ferreira, H (2020) Extended-spectrum beta-lactamase (ESBL)producing Enterobacteriaceae in cattle production - a threat around the world. Heliyon 6: e 03206.

Rasheed MU, Thajuddin N, Ahamed P, Teklemariam Z, Jamil K (2014) Antimicrobial drug resistance in strains of E.coli isolated from food sources. Rev Inst Med Trop Sao Paulo 56: 341-346

Shome BR, Das Mitra S, Bhuvana M, Krithiga N, Velu D, Shome R, Isloor S, Barbuddhe SB, Rahman H (2011) Multiplex PCR assay for species identification of bovine mastitis pathogens. J Appl Microbiol 111: $1349-1356$

Singh R, Schroeder CM, Meng J, White DG, McDermott PF, Wagner DD, Yang H, Simjee S, DebRoy C, Walker RD, Zhao S (2005) Identification of antimicrobial resistance and class 1 integrons in Shiga toxinproducing $E$. coli recovered from humans and food animals. J Antimicrob Chem 56:216-219

Soomro AH, Arain MA, Khaskheli M, Bhutto B (2002) Isolation of E. coli from raw milk and milk products in relation to public health sold under market conditions at Tandojam. Pak J Nutr 1: 151-152

Tadesse DA, Zhao S, Tong E, Ayers S, Singh A, Bartholomew MJ, McDermott PF (2012) Antimicrobial drug resistance in E. coli from humans and food animals, United States, 1950-2002. Emerg Infect Dis 18: 741

Thaker HC, Brahmbhatt MN, Nayak JB, Thaker HC (2013) Isolation and identification of Staphylococcus aureus from milk and milk products and their drug resistance patterns in Anand, Gujarat. Vet World 6: $10-13$

Torpdahl M, Nielsen EM, Scheutz F, Olesen B, Hansen DS, Hasman H (2013) Detection of a Shiga toxin- and extended-spectrum- $\beta$ lactamase-producing E. coli $\mathrm{O} 157: \mathrm{H} 7$ human clinical isolate. $\mathrm{J}$ Antimicrob Chemother 68: 1203-1204

Van Hoek AHAM, Veenman C, van Overbeek WM, Lynch G, de Roda Husman AM, Blaak H (2015) Prevalence and characterization of ESBL- and AmpC-producing Enterobacteriaceae on retail vegetables. Int J Food Microbiol 204: 1-8

Weill FX, Demartin M, Tandé D, Espié E, Rakotoarivony I, Grimont PA (2004) SHV-12-like extended-spectrum- $\beta$-lactamase-producing strains of Salmonella enterica serotypes Babelsberg and Enteritidis isolated in France among infants adopted from Mali. J clin Microbiol 42: $2432-2437$ 